\title{
Yersinia enterocolitica septicaemia from transfusion of red cell concentrate stored for 16 days
}

\author{
B L Jones, M H Saw, M F Hanson, M J Mackie, J Scott, W G Murphy
}

\begin{abstract}
Two cases of transfusion transmitted Yersinia enterocolitica biotype 3, serotype 09 infection occurred in south east Scotland within four months of each other. In one case, a 79 year old man died the day after receiving a unit of red cell concentrate that had been stored for 29 days after donation. In the second case a 78 year old man died three days after transfusion of a unit of red cell concentrate that had been collected 16 days before transfusion. The donors of both units had no symptoms attributed to gastrointestinal infection. Early outdating of blood for transfusion after three weeks of storage is unlikely to eradicate $Y$ enterocolitica associated fatalities from blood transfusion, and alternative methods should be considered.
\end{abstract}

( Clin Pathol 1993;46:477-478)

Transfusion of blood contaminated with bacteria is rare, but carries a high mortality. In recent years the commonest organism implicated in deaths caused by transfusion induced septicaemia or endotoxinaemia has been Yersinia enterocolitica. Although this predominance may be influenced by reporting bias, the incidence of contamination of blood for transfusion by this organism has probably increased. Twenty six cases worldwide had been reported by 1991 since the first report in 1982. ${ }^{1-5}$ Until now all fatalities associated with transfusion of blood contaminated with $Y$ enterocolitica were caused by the transfusion of blood that had been stored for 21 days following donation, or longer. This observation is consistent with in vitro data, which showed that small inocula of $Y$ enterocolitica inoculated into whole blood could not be recovered until the second week of incubation. ${ }^{6}$ Contamination of a blood donation with $Y$ enterocolitica is probably always due to bacteraemia in the donor at the time of donation. ${ }^{1}$ The bacteraemia is asymptomatic in about half of the donors.

During the last four months of 1991 two cases of transfusion-transmitted $Y$ enterocolitica septicaemia occurred in the south east of Scotland. Both recipients died within three days of transfusion. The same strain of $Y$ enterocolitica was implicated in both cases. The donors were from different parts of the region, and shared no other identifiable common factors. Both were asymptomatic. One patient received a unit that had been stored for 29 days before transfusion, and died the following day. The other patient received a unit of red cell concentrate that had been stored for 16 days since donation. This case is described in detail.

\section{Case report}

A 78 year old man with chronic lymphatic leukaemia and chronic renal failure was transfused with red cell concentrate for anaemia. When about $100 \mathrm{ml}$ of the first unit had been transfused the patient become hypotensive and febrile (blood pressure $85 / 60 \mathrm{~mm} \mathrm{Hg}$, temperature $40^{\circ} \mathrm{C}$ ) with rigours, nausea, and diarrhoea. The transfusion was stopped and serological incompatibility was excluded. The blood unit did not appear discoloured at this point. Blood samples for bacterial culture were taken from the patient and from the donor unit. Treatment was begun immediately with ciprofloxacin and gentamicin. Forty eight hours later, the patient was normotensive and afebrile with good urine output, but after a further 24 hours he developed acute pulmonary oedema and died. Post mortem examination showed the immediate cause of death to be myocardial ischaemia. The initial blood cultures from patient and donor unit grew $Y$ enterocolitica, biotype 3, serotype 09 (Central Public Health Laboratory, Colindale, London), sensitive to ciprofloxacin and gentamicin. Post mortem culture of splenic tissue was negative for $Y$ enterocolitica. The donor of the red blood cell unit could recall no symptoms of gastrointestinal upset during the weeks before donation, and had been well following donation. No other blood component from that donation had been transfused. Serum from the donor was tested for the presence of agglutinating antibodies to $Y$ enterocolitica (serotype 09). Samples were available from serum archives for two donations: four months before the implicated donation, and the implicated donation, and from follow up testing at four, eight, and 12 months after the implicated donation. The earliest sample contained no clinically relevant agglutinating antibodies; antibodies were present to a titre of $1 / 1280$ in serum from the implicated donation, and to titres of $1 / 160$, $1 / 160$, and $1 / 80$ at four, eight, and 12 months afterwards, respectively. Serum from the donation implicated in the second case was also tested: agglutinating antibodies were present to a titre of $1 / 1280$. 


\section{Discussion}

Since these cases, no further episodes of $Y$ enterocolitica contamination of donor blood have been noted in this region. No cases of gastroenteritis due to $Y$ enterocolitica have been notified to the public health department. This suggests that the temporal association of the two cases was due to chance and was not the start of a local outbreak. While still rare, the contamination of blood donations by $Y$ enterocolitica is a relatively recent observation, and suggests that the incidence in the donor population has increased or that the detection rate has increased in the presence of a constant rate of contamination. Alternatively, changes in blood transfusion practice, such as the use of red cell concentrates or additive solutions in intensively plasma reduced blood units, may favour the growth of this micro-organism in stored blood.

Several strategies to reduce the risk of morbidity from $Y$ enterocolitica contamination of blood donations have been proposed, ${ }^{1}$ including exclusion of donors with a recent history of gastrointestinal upset, outdating of blood donations after three weeks of storage, testing of blood for the presence of endotoxin before release, or filtration of blood to remove leucocyte engulfed or adherent bacteria. Only about half of the contaminated units will be screened out by donor exclusion techniques, ${ }^{1}$ and as indicated in this report, early outdating of stored blood after three weeks of shelf life will not eradicate serious morbidity. Recent studies indicate that leucocyte depletion of donated blood may remove leucocyte engulfed bacteria, ${ }^{7}$ although whether this will result in an overall improvement in the bacteriological safety of stored blood still needs to be established. ${ }^{8}$
Management of severe acute transfusion reactions that could be due to bacterial contamination of the transfused blood unit may be complicated by the fact that considerable growth of $Y$ enterocolitica can be present without visible haemolysis or discolouration, ${ }^{9}$ as occurred in this case. Rapid administration of antibiotics with activity against Gram positive and Gram negative organisms resulted in a successful initial resusitation in the case reported here: it is necessary to assume that septicaemia has occurred when there is no evidence of serological mismatch on rapid rechecking, and before bacteriological confirmation has been obtained.

Tipple MA, Bland LA, Murphy J, et al. Sepsis associated with transfusion of red cells contaminated with Yersinia enterocolitica. Transfusion 1990;30:207-13.

2 Stubbs JR, Reddy RL, Elg SA, Perry EH, Adcock LL, McCullough J. Fatal Yersinia enterocolitica (serotype $0: 5,27$ ) sepsis after blood transfusion. Vox Sang 1991;61:18-23.

3 Prentice M, Cope D, Weinbren M, O'Driscoll J. Infectious complications of blood transfusion. $B M^{\top}$ 1990;300:678-9.

4 Smillie J, Ala F. Fatal transfusion-associated Yersinia enterocolitica infection. Transfusion Med 1991;1 (Suppl 2):80.

5 Ferguson K, Macvarish I, Barr A, Mitchell R. Yersiniaanother hazard of transfusion? Transfusion Med 1991;1 (Suppl 2):80.

6 Ardiuno MJ, Bland LA, Tipple MA, Aguero SM, Faver MS, Jarvis WR. Growth and endotoxin production of Yersinia enterocolitica and Enterobacter agglomerans in packed erythrocytes. F Clin Microbiol 1989;27:1483-9.

7 Pietersz RNI, Reesink HW, Pauw W, Dekker WJA Buisman L. Prevention of Yersinia enterocolitica growth in red-blood-cell concentrates. Lancet 1992;340:755-6.

8 Gibb AP, Martin KM, Davidson GA, Walker B, Murphy WG. Bacterial growth in blood for transfusion. Lancet 1992;340:1222-3.

9 Anonymous. Yersinia entercolitica bacteremia and endotoxin shock associated with red blood cell transfusionWeekly Report 1988;37:577-8. 